LA-UR-99-3322

Approved for public release; distribution is unlimited.

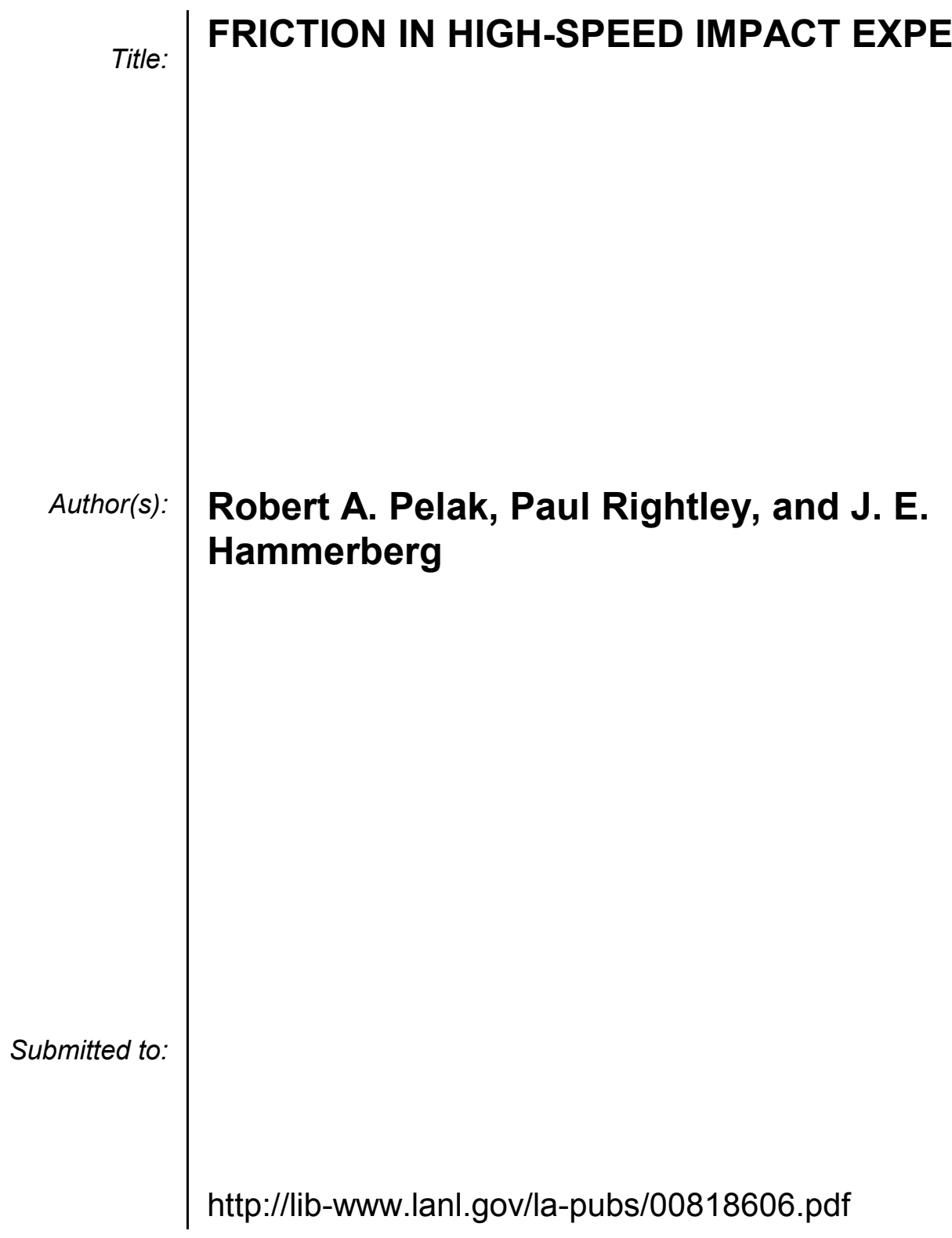

Los Alamos National Laboratory, an affirmative action/equal opportunity employer, is operated by the University of California for the U.S. Department of Energy under contract W-7405-ENG-36. By acceptance of this article, the publisher recognizes that the U.S. Government retains a nonexclusive, royaltyfree license to publish or reproduce the published form of this contribution, or to allow others to do so, for U.S. Government purposes. Los Alamos National Laboratory requests that the publisher identify this article as work performed under the auspices of the U.S. Department of Energy. Los Alamos National Laboratory strongly supports academic freedom and a researcher's right to publish; as an institution, however, the Laboratory does not endorse the viewpoint of a publication or guarantee its technical correctness. 


\title{
FRICTION IN HIGH-SPEED IMPACT EXPERIMENTS
}

\author{
Robert A. Pelak ${ }^{a}$ Paul Rightley $^{a}$, and J. E. Hammerberg ${ }^{b}$ \\ ${ }^{a}$ Dynamic Experimentation Division (MS P940) and \\ ${ }^{b}$ Applied Theoretical and Computational Physics Division (MS F664) \\ Los Alamos National Laboratory, Los Alamos, NM 87545
}

\begin{abstract}
The physical interactions at the contact interface between two metals moving relative to one another are not well understood, particularly when the relative velocity between the bodies becomes a significant fraction of the sound speed in either material. Our goal is to characterize the interfacial dynamics occurring between two metal surfaces sliding at high loads (up to $300 \mathrm{kbar}$ ) and at high speeds (greater than $100 \mathrm{~m} / \mathrm{s}$ ). We are developing a technique where a high-speed spinning projectile is fired from a rifled gun at a rod instrumented with electrical resistance strain gauges for measuring both longitudinal and torsional strain waves. The observed traces, in conjunction with computer simulations, are used to estimate the normal and tangential force components at the interface to produce an estimate of the coefficient of friction. A preliminary estimate for a copper/steel interface is presented.
\end{abstract}

Studies of sliding friction under conditions of high relative speed (over $100 \mathrm{~m} / \mathrm{s}$ ) are very limited [1-4]. The primary result of these investigations has been to observe that the substantial amount of frictional heat can lead to softening and melting of one of the sliders. This produces a decrease in the coefficient of friction with increasing relative speed until hydrodynamic drag becomes sufficient to cause the coefficient to increase with sliding speed [2]. Based upon an idea by Krafft [5], we are developing a new technique to perform dynamic measurements of sliding friction for high normal loading as well as high sliding speeds.

A copper projectile was fired from a powder gun at a stationary target rod located approximately four meters away. Rifling in the gun barrel gave the projectile rotational as well as translational kinetic energy. Upon impact the rotating projectile slid against the circular face of the rod while its momentum produced a substantial normal load. The diameters of both the projectile and the target were $14.3 \mathrm{~mm}$. The impact surfaces of both the projectile and the target were prepared by sanding with increasing grades of abrasive paper to a maximum of 400 grit. The projectile speed was measured using two light screens located between the gun and target. The alignment and timing of the impact was verified with images taken by a Imacon 468 CCD camera system. The target rod was instrumented with two sets of electronic resistance strain gauges. Each gauge set consisted of six strain gauges wired into two separate Wheatstone bridge circuits: two for longitudinal (or axial) strain waves and four for torsional strain waves. Fast response $(-3 \mathrm{~dB}$ at $500 \mathrm{MHz})$ amplifiers provided signals for a digital storage oscilloscope. Initially, the gauge sets were located 18 and 36 inches from the impact site. Since this work is still in the technique development stage, target rods are used in multiple tests. However, before using a target rod in a second or third shot, the leading several inches of the rod 
were cut off to remove portions that had undergone appreciable plastic deformation.

Figure 1 shows four sequential images of an impact taken with the Imacon 468 camera. The images were taken at $2 \mu$ s intervals with a $1 \mu$ s exposure. The 110 grade copper projectile impacted the 304 stainless steel rod with a longitudinal velocity of $1023 \mathrm{~m} / \mathrm{s}$ and an azimuthal velocity of $138 \mathrm{~m} / \mathrm{s}$ at the outer edge. Each image consists of two views of the impact. The lower portion of the image is a side view as directly seen by the camera; the upper portion is a top view taken with the aid of a mirror oriented at 45 degrees and placed above and behind the rod. Examination of the target rod after the experiment showed that it underwent significant amounts of fracture and deformation as a result of the impact. The copper projectile was totally destroyed by the impact; images taken subsequent to those shown in Fig. 1 indicate that destruction occurred approximately $40 \mu \mathrm{s}$ following impact.

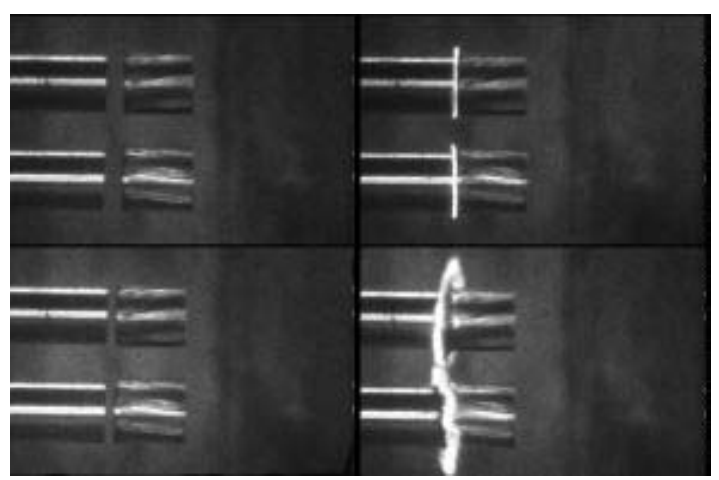

FIGURE 1. 110 grade copper projectile impact on 304 stainless steel target rod.

Figures 2 and 3 show the time dependent strains (longitudinal and torsional, respectively) seen by the forward and rear gauge sets. All four sets of gauges recorded the passage of strain pulses produced by the impact. Long (6 foot) target rods were used so that the slower torsion pulses would arrive at both gauge sets prior to the reflected longitudinal pulses (the positive going peaks in Fig. 2). The longitudinal gauges recorded a strain pulse with a "sawtooth" shape. This shape differs substantially from the square wave seen in other tests where the impact was less severe and the rod's response was purely elastic. The sharp rise and fall recorded in the tor-

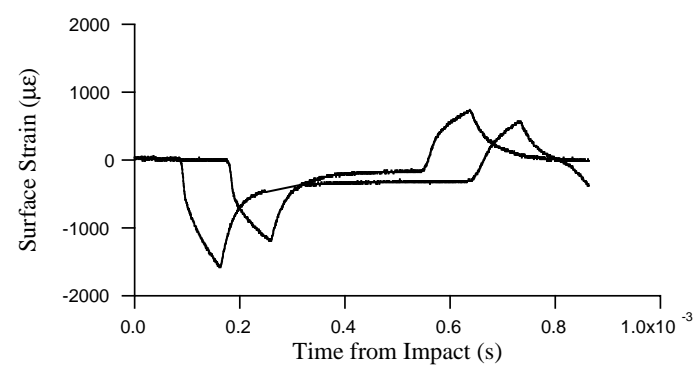

FIGURE 2. Longitudinal strain traces from impact; forward and and rear gauges.

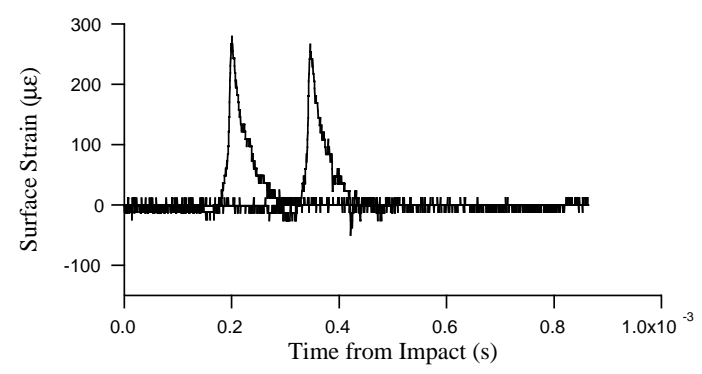

FIGURE 3. Torsional strain traces from impact; forward and rear gauges.

sional signals and in the purely elastic tests indicate that the sawtooth shape is not the result of limited electronic response time. The longitudinal strain traces indicate the presence of some residual strain following the decay of the pulse; this residual strain decreases with the passage of each reflected pulse. No residual strain is apparent in the torsional strain traces.

From their leading edges, the velocities of the longitudinal and torsional pulses as they travel between the two sets of gauges can be determined. For the data shown in Figs. 2 and 3, these speeds correspond to 5.07 and $3.04 \mathrm{~mm} / \mu \mathrm{s}$, respectively. Impacts of this type were performed several times producing mean speeds of $5.06 \pm .02$ and $3.08 \pm .03 \mathrm{~mm} / \mu \mathrm{s}$, respectively. The longitudinal sound speed was measured to be $4.93 \mathrm{~mm} / \mu$ s from analysis of the less severe elastic impacts mentioned earlier. A similar measurement of the torsional sound speed in 304 stainless steel was not possible, but has been reported in the literature as $3.12 \mathrm{~mm} / \mu \mathrm{s}$ [6]. We can conclude that the longitudinal pulse is traveling at slightly supersonic speeds and is a weak shock while the torsional pulse is strictly elastic. 
The propagation speed of the strain pulses between the impact site and the forward set of strain gauges can be evaluated in a similar manner. For the traces shown in Figs. 2 and 3 these speeds are 5.11 and $2.49 \mathrm{~mm} / \mu \mathrm{s}$, respectively. The reduced value for the torsion pulse speed clearly indicates that the deformation of material near the impact site has an effect on its propagation speed.

We have considered some possible experimental sources for the origin of the sawtooth shape of the longitudinal strain pulses. Due to the distance between the gun barrel and target surface, we did not always achieve such nearly perfect aim between projectile and target as shown in Fig. 1. Projectiles would occasionally impact the target up to $3 \mathrm{~mm}$ off center. Figure 4 shows the longitudinal strain traces from the rear gauge set for three shots fired under similar conditions; the one shown in Fig. 2 and two others with impacts off center by distances of 1 and $3 \mathrm{~mm}$. The impact velocities were nearly identical $(1023,1030$, and $1035 \mathrm{~m} / \mathrm{s})$. The traces from these three impacts are nearly indistinguishable. With this in mind, it seems unlikely that that the sawtooth shape of these pulses is due to aiming error. It is possible that the two impacting surfaces were not perfectly parallel and that this contributed to the sawtooth pulse shape; however, such a deviation must be extremely small: less than $0.6^{\circ}$ based upon pixel resolution. It would also have to be repeated with very high precision to produce the three identical traces of Fig. 4. In our judgement, a nonplanar impact is probably not the sole origin of the sawtooth shape.

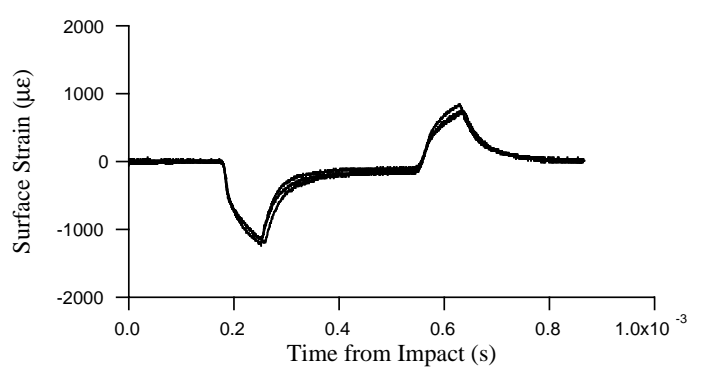

FIGURE 4. Longitudinal strain traces from three impacts.

From the definitions of the appropriate elastic moduli, we can derive an approximation for the co- efficient of friction, $\mu$

$$
\mu \approx \frac{\sigma_{z \theta}}{\sigma_{z z}} \approx \frac{2 G}{B+\frac{4}{3} G} \frac{\varepsilon_{z \theta}}{\varepsilon_{z z}}=\frac{2}{\frac{B}{G}+\frac{4}{3}} \frac{\varepsilon_{z \theta}}{\varepsilon_{z z}},
$$

where $\sigma_{z z}$ and $\sigma_{z \theta}$ are the normal and shear stresses, $\varepsilon_{z z}$ and $\varepsilon_{z \theta}$ are the normal and shear strains, and $B$ and $G$ are the bulk and shear moduli. For 304 stainless steel, the ratio $B / G$ is approximately 2.1 . Using the peak values of the longitudinal and torsional pulses, we can estimate the coefficient of friction:

TABLE 1. Measured estimates of the coefficient of friction.

\begin{tabular}{lll} 
Impact Vel. & Max. Sliding Vel. & $\mu$ \\
\hline \hline $1095 \mathrm{~m} / \mathrm{s}$ & $148 \mathrm{~m} / \mathrm{s}$ & 0.055 \\
$1023 \mathrm{~m} / \mathrm{s}$ & $138 \mathrm{~m} / \mathrm{s}$ & 0.073 \\
$897 \mathrm{~m} / \mathrm{s}$ & $121 \mathrm{~m} / \mathrm{s}$ & 0.023
\end{tabular}

The uncertainties in $\mu$ cannot be meaningfully quantified because of issues surrounding several significant assumptions. First, there is the assumption that the longitudinal and torsional waves are equally attenuated as they pass along the rod. The traces shown in Figs. 2 and 3 show that this is clearly not the case, as the longitudinal pulses attenuate significantly while the torsional pulses do not. To partially account for this, we determined the rate of attenuation from a number of shots performed with the strain gauges located at varying distances down the rod. The longitudinal peak height observed in an experiment was then scaled using the attenuation rate to determine what its value would be at the impact site; this scaled value was used in Eq. 1. This approach is only truly valid for portions of the rod that have not been plastically deformed, but we are applying it to the entire distance between the impact site and the gauge.

Another assumption concerns the use of peak values of the strain in the equation for $\mu$. By doing this, we are assuming that these peak values are dynamically correlated in that they reflect sliding conditions at the same instant of time. The traces of Figs. 2 and 3 suggest that this is not true; the peak value of the longitudinal pulse occurs about $75 \mu$ s following its leading edge while this difference is about $25 \mu \mathrm{s}$ for the torsional pulse. Still, it is possible that the two peaks are better correlated than they appear because the apparent reduced speed of the torsional 
wave through the deformed region could be masking a delay in the start of production of significant torsional strain.

The values shown in Table 1 are somewhat lower than those measured in previous studies. Bowden and Persson [2] measured values of approximately 0.20 for $\mu$ at these speeds by dropping a rotating steel ball on a copper plate. Consequently, these measurements were conducted at considerably lower normal loads and were integrated over longer time scales. At speeds of several hundred meters per second they also found a tendency for $\mu$ to decrease as the load is increased [1,2]; however, they did not explore that phenomenon at speeds close to those used in this study. Montgomery [3] also studied high speed sliding of copper on gun steel using a pin-on-disk technique. He also observed values for $\mu$ greater than ours, but he too averaged over longer sliding periods of time than we have. Additionally, the pin-on-disk technique constantly supplies fresh surfaces for sliding, potentially leading to other effects that may alter the sliding behavior.

The large amount of plastic deformation near the impact surfaces can affect the shape of the strain waves that emanate from it. For instance, the copper projectile was observed to be destroyed within 40 microseconds of impact but the longitudinal strain pulse is over twice as long. To better understand this, we have performed some simulations of the impact using a Lagrangian hydrocode. The limitations of the code to two dimensions did not allow us to consider projectile rotation or torsional strain pulses. These simulations reveal that an impact such as we have performed should produce a slightly supersonic strain pulse of which about $2 \%$ of the total strain is plastic strain. The total strain is approximately (within a factor of 2) equal to the peak strain observed in our experiments; similarly, the computed plastic strain is within a factor of 5 of the residual strain observed in the longitudinal strain trace. One significant difference is that the stain pulse has a flat top profile like that in a purely elastic collision. The origin of this discrepancy is currently not understood and will be a subject of further investigation. Three dimensional Lagrangian hydrocodes are currently under development that will allow for study of the torsional pulses as well as the longitudinal ones.

\section{REFERENCES}

1. Bowden, F. P., and Frietag, E. H., Proc. Roy. Soc. A 248, 350-367 (1958).

2. Bowden, F. P., and Persson, E. H., Proc. Roy. Soc. A 260, 433-458 (1961).

3. Montgomery, R. S., Wear 36, 275-298 (1976).

4. Rajagopalan, S., Irfan, M. A., and Prakash, V., Wear 225-9, 1222-37 (1999).

5. Krafft, J. M., J. Appl. Phys. 26, 1248-1253 (1955).

6. Marsh, S., LASL Shock Hugoniot Data, University of California Press, Berkeley CA, 1980, p. 212. 